\title{
Application of MISO Volterra Series for Modeling Subharmonic of Ultrasound Contrast Agent
}

\author{
C. Samakee and P. Phukpattaranont
}

\begin{abstract}
To overcome the problem that single input single output (SISO) Volterra cannot model subharmonic components of ultrasound contrast agent (UCA), we propose the multiple-input-single-output (MISO) Volterra in this paper. The Volterra kernel estimations are carried out using singular value decomposition (SVD) method from numerical simulation data of representative of subharmonic based on the Church's model. The performance of the method is evaluated using the normalized mean-square errors (NMSE) for the first-, secondand third-order Volterra model. Accuracy of the outputs from Volterra model is shown both in time domain and frequency domain. The resulting estimation from the third-order MISO Volterra model provides the best achievable improvement at the NMSE of -12.01 dB for 0.6 MPa excitation pressure (optimal of subharmonic generation).
\end{abstract}

Index Terms-Ultrasound contrast agent, microbubble, volterra series, subharmonic, nonlinear oscillation.

\section{INTRODUCTION}

Ultrasound contrast agents (UCAs) consist of encapsulated gas microbubbles with typical diameters of a few microns that are injected into blood. UCAs are used in ultrasound medical applications as contrast agents to enhance backscatter signals between blood and surrounding tissues for improving the quality of ultrasound images [1]-[3]. The behavior of UCAs depends on the amplitude levels of the acoustic pressure of incident ultrasound wave. Additionally, the excitation pressure input at a transmit frequency of $f_{0}$ can enable the response signals consisting of both higher harmonic $\left(2 f_{0}, 3 f_{0}\right.$, etc. $)$ and subharmonic $\left(f_{0} / 2\right)$ components [4]. To employ the second harmonic generation from UCAs, the harmonic imaging (HI; $2 f_{0}$ ) has been developed [5]. However, the second harmonic generation and accumulation in tissue may lead to the tradeoff in contrast resolution [6].

Under specific conditions, UCAs can generate subharmonic components and imaging modality based on subharmonics can be employed. The advantage of subharmonic imaging (SHI; $f_{0} / 2$ ) compared to $\mathrm{HI}$ is that tissue signal is minimal. This results in the potential to produce higher contrast-to-tissue rations (CTR). Reports have described the feasibility and implementation of SHI [6-8]. To improve the subharmonic generation from UCAs the knowledge about the description of acoustic properties of

Manuscript received April 16, 2012; revised May 9, 2012. This work was financially supported by Department of Electrical Engineering, Faculty Engineering, Prince of Songkla University, Thailand

The authors are with the Department of Electrical Engineering, Prince of Songkla University, Thailand (e-mail: chindasamakee@hotmail.com, pornchai.p@psu.ac.th).
UCAs is necessary and needed. Researchers have been developed the equation for modeling free gas microbubble oscillation based on the relationship between the physical parameters of the encapsulated microbubble and the excitation pressure as reported in [9-10]. For example, the subharmonic simulations resulting from the Church's equation are shown to be in agreement with those from the experimental results [11].

The equation models enable the simulation of UCA oscillation behavior but they cannot be employed as a harmonic separation model for imaging purposes. As a result, the nonlinear model based on the Volterra series is introduced. Volterra series can be used as an appropriate mathematical model for UCA nonlinear oscillation and a harmonic separation model for imaging purposes. The model for describing the nonlinear oscillation of UCAs based on single-input-single-output (SISO) Volterra series was given in [12]. The application of Volterra series based on prediction model for improving the quality of ultrasound imaging was presented in [13]. However, SISO Volterra series cannot model subharmonic oscillation systems.

To use Volterra series as a model for subharmonic oscillation systems, some modifications of input signals are required and the multiple-input-single-output (MISO) Volterra series is introduced [14]-[15]. The main purpose of this paper is to apply MISO Volterra series for modeling subharmonic oscillation from UCA. The identification of Volterra kernels can be solved numerically a system of linear equations. Results from the model based on MISO Volterra series provide another ways for the understanding of UCA subharmonic generation. This may lead to a new imaging modality, which utilizes the subharmonic separation based MISO Volterra series.

The rest of this paper is organized as follows. Section II presents the Volterra system representation and the identification method for determining the coefficients of Volterra series. The identification details of a third-order MISO Volterra model with simulation data obtained from the Church's equation are given in Section III. Comparison results between the output of Church's equation and the predicted output from the third-order MISO Volterra model at different excitation amplitudes and series lengths are shown in Section IV. Finally, we summarize and discuss our finding in Section V.

\section{VOLTERRA SYSTEM}

\section{A. Volterra Series Expansion}

The Volterra series expansion is widely employed to 
represent the input/output description for nonlinear systems analysis. The notation of Volterra series was first introduced by the Italian mathematician Vito Volterra. An example of Volterra theory application was nonlinear circuit analysis by the mathematician Norbert Wiener [16]. The theory states that any time-invariant systems can be modeled with the input-output relationship as,

$$
\begin{aligned}
& y(t)=h_{0}+\int_{-\infty}^{\infty} h_{1}\left(\tau_{1}\right) x\left(t-\tau_{1}\right) d \tau_{1} \\
& +\int_{-\infty}^{\infty} \int_{-\infty}^{\infty} h_{2}\left(\tau_{1}, \tau_{2}\right) x\left(t-\tau_{1}\right) x\left(t-\tau_{2}\right) d \tau_{1} d \tau_{2}+\ldots \\
& +\int_{-\infty}^{\infty} \int_{-\infty}^{\infty} \ldots \int_{-\infty}^{\infty} h_{k}\left(\tau_{1}, \tau_{2}, \ldots, \tau_{k}\right) x\left(t-\tau_{1}\right) x\left(t-\tau_{2}\right) \ldots x\left(t-\tau_{k}\right) d \tau_{1} d \tau_{2} \ldots d \tau_{k}+\ldots,
\end{aligned}
$$

where $y(t)$ denotes the response of predicted model, $x(t)$ denotes the excitation of the system, $h_{k}\left(\tau_{1}, \tau_{2}, \ldots ., \tau_{k}\right)$ is the $k$ th-order Volterra kernel and $\tau_{k}$ is the system memory. Alternatively, the representation of causal discrete-time Volterra series can be expressed as [17]

$$
\begin{aligned}
y(n)= & h_{0}+\sum_{\tau_{1}=0}^{N-1} h_{1}\left(\tau_{1}\right) x\left(n-\tau_{1}\right) \\
& +\sum_{\tau_{1}=0}^{N-1} \sum_{\tau_{2}=0}^{N-1} h_{2}\left(\tau_{1}, \tau_{2}\right) x\left(n-\tau_{1}\right) x\left(n-\tau_{2}\right) \ldots \\
& +\sum_{\tau_{1}=0}^{N-1} \ldots \sum_{\tau_{k}=0}^{N-1} h_{k}\left(\tau_{1}, \ldots, \tau_{k}\right) x\left(n-\tau_{1}\right) \ldots x\left(n-\tau_{k}\right),
\end{aligned}
$$

where $y(n)$ and $x(n)$ denote the input and output data sequence, respectively, $N$ is the number of samples memory of $k$ th-order truncated Volterra series. A $k$ th-order Volterra kernel is bounded-input bounded-output (BIBO) stable if

$$
\sum_{\tau_{1}=0}^{N-1} \ldots \sum_{\tau_{k}=0}^{N-1} \mid h_{k}\left(\tau_{1}, \ldots, \tau_{k} \mid<\infty\right.
$$

For kernels length of samples identified in (2), the number of all coefficients in kernels can be calculated as

$$
K=\sum_{i=1}^{k}\left(\begin{array}{c}
N+i-1 \\
i
\end{array}\right)
$$

where $k$ is the system order and if it is excessively large, the system memory $K$ will be increase.

\section{B. System Identification}

In system identification, the relation between the response $y(n)$ of the model and its input $x(n)$ in (2), can be written in the matrix form

$$
\mathbf{y}=\mathbf{X h}
$$

The output vector $\mathbf{y}$ of the system is defined as

$$
\mathbf{y}=(y(0) \quad y(1) \ldots y(n))^{T},
$$

where the input matrix $\mathbf{X}$ is defined as:

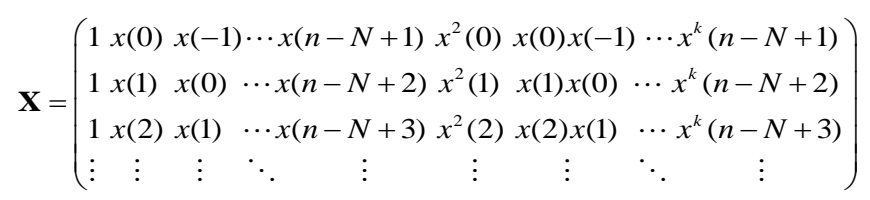

and the coefficient vector $\mathbf{h}$ is defined as:

$$
\begin{aligned}
\mathbf{h}= & \left(h_{0} h_{1}(0) \ldots h_{1}(N-1) h_{2}(0,0) h_{2}(0,1) \ldots\right. \\
& \left.h_{k}(N-1, \ldots, N-1)\right)^{T} .
\end{aligned}
$$

Then, the Volterra kernel of a system is calculated with the following equation:

$$
\mathbf{h}=(\mathbf{X})^{-1} \mathbf{y}
$$

The solution of a linear system of equations can be written using a singular value decomposition (SVD) of $\mathbf{X}$, which is defined as [18]

$$
\mathbf{X}=\mathbf{U} \Sigma \mathbf{V}^{T}=\sum_{i=1}^{r} \sigma_{i} \mathbf{u}_{i} \mathbf{v}_{i}^{T}
$$

where $\mathbf{U}$ is an $m \times m$ orthonormal matrix $\mathbf{V}$ is an $n \times n$ orthonormal matrix and $\sum$ is an $m \times n$ diagonal matrix. $\sigma=$ $\operatorname{diag}\left(\sum\right)$ are the singular value sorted in descending order $\sigma_{1}$ $\geq \sigma_{2} \geq \ldots \geq \sigma_{\mathrm{n}} \geq 0$. The solution is then given by

$$
\mathbf{h}=\left(\mathbf{U} \sum \mathbf{V}^{T}\right)^{-1} \mathbf{y}=\sum_{i=1}^{n} \mathbf{v}_{\mathrm{i}} \frac{1}{\sigma_{i}} \mathbf{u}_{\mathrm{i}}^{T} \mathbf{y}
$$

The SVD of $\mathbf{X}$ forms a basis for regularization. The truncated SVD (TSVD) regularization can be obtained when the singular values are modified with the condition

$$
\sum_{i i}^{-1}=\left\{\begin{array}{lll}
\frac{1}{\sigma_{i}} & \text { if } & \sigma_{i} \geq \alpha \\
0 & \text { if } & \sigma_{i}<\alpha
\end{array}\right.
$$

where $\alpha$ is a certain threshold. The selected threshold value is optimized solution that is in minimum norm least squares of the estimation error, i.e., $\min \|\mathbf{y}-\mathbf{X h}\|^{2}$.

\section{METHODS}

\section{A. Simulation of Subharmonic generation}

Subharmonic data were obtained from the simulation of microbubble oscillation behavior, which were numerical solutions of the Church's equation given by [10]

$$
\begin{array}{r}
\rho_{\mathrm{L}}\left(R^{\prime \prime} R+\frac{3}{2}\left(R^{\prime}\right)^{2}\right)=p_{0}\left(\left(\frac{R_{0}}{R}\right)^{3}-1\right)-P_{i n}(t)-4 \mu_{\mathrm{L}} \frac{R^{\prime}}{R} \\
-12 \mu_{\mathrm{S}} \frac{d_{\mathrm{S}} R_{0}^{2}}{R^{3}} \frac{R^{\prime}}{R}-12 G_{s} \frac{d_{s} R_{0}^{2}}{R}\left(1-\frac{R_{0}}{R}\right),
\end{array}
$$


where $R$ is the instantaneous radius of the microbubble, $R^{\prime}, R^{\prime \prime}$ are the first and second time derivatives of instantaneous radius, respectively. The parameter terms in (11) are defined in the Table I. The $P_{i n}(t)=p \cos (\omega t)$ is a sinusoidal pulse of the incident excitation amplitude pressure $(p)$ at a frequency of $\omega \mathrm{rad} / \mathrm{s}$. The resonance frequency $\left(f_{0}\right)$ of the encapsulated microbubble is

$$
f_{0}=\frac{\omega_{0}}{2 \pi}=\frac{1}{2 \pi R_{0}} \sqrt{\frac{1}{\rho_{\mathrm{L}}}\left(3 \kappa p_{0}+12 G_{\mathrm{S}} \frac{d_{\mathrm{S}}}{R_{0}}\right)} .
$$

The acoustic pressure at a distance of $r=0.6 \mathrm{~cm}$ from the center of the microbubble can be expressed as

$$
P_{\text {out }}(t)=\rho_{\mathrm{L}} r^{-1}\left(R^{2} R^{\prime \prime}+2 R\left(R^{\prime}\right)^{2}\right)
$$

The excitation pressure has a frequency at twice of the resonance frequency $\left(2 f_{0}=4.5 \mathrm{MHz}\right)$ with a time duration of $40 \mu \mathrm{s}$. This excitation was shown to be optimized for subharmonic generation as presented in [19]. The sampling frequency was at $60 \mathrm{MHz}$. The solution of (11) is computed by using a fourth-order Runge-Kutta algorithm with the initial conditions $R=R_{0}$ and $R^{\prime}=0$ at time $t=0$.

\section{B. MISO Volterra Series Identification}

The solutions from the Church's model are used in the identification of a third-order MISO Volterra series, which is given by

$$
\begin{aligned}
& \hat{y}(n)= h_{0}^{\text {栥 }}+\sum_{\tau_{1}=0}^{N-1} h_{1}\left(\tau_{1}\right) x\left(n-\tau_{1}\right) \\
&+\sum_{\tau_{1}=0}^{N-1} \sum_{\tau_{2}=0}^{N-1} \hat{h}_{2}\left(\tau_{1}, \tau_{2}\right) x\left(n-\tau_{1}\right) x\left(n-\tau_{2}\right) \\
&+\sum_{\tau_{1}=0}^{N-1} \sum_{\tau_{2}=0}^{N-1} \sum_{\tau_{3}=0}^{N-1} \hat{h}_{3}\left(\tau_{1}, \tau_{2}, \tau_{3}\right) x\left(n-\tau_{1}\right) x\left(n-\tau_{2}\right) x\left(n-\tau_{3}\right),
\end{aligned}
$$

where $\hat{y}(n)$ is the predicted output from Volterra series, $\hat{h}_{0}, \hat{h}_{1}\left(\tau_{1}\right), \hat{h}_{2}\left(\tau_{1}, \tau_{2}\right)$ and $\hat{h}_{3}\left(\tau_{1}, \tau_{2}, \tau_{3}\right)$ are the coefficients of the zero-order, first-order, second-order, and third-order Volterra kernels, respectively. $x(n)$ denotes the acoustic pressure input signal. The SISO Volterra series cannot model subharmonics because the Volterra series responds to a periodic excitation with a periodic signal with the same period. However, if the input and output signals are modified to have the period $T$, which corresponding to the subharmonic period, the Volterra series could be used for modeling the sub harmonics oscillation by the MISO method [14]-[15]. The input and output signals $P_{\text {in }}$ and $P_{\text {out }}$ are decomposed to $p$-dimensional as $P_{i n(\bmod )}=\left[x_{1} ; \ldots ; x_{\mathrm{i}} ; \ldots ; x_{p}\right]$ and $P_{\text {out }(\mathrm{mod})}=\left[y_{1} ; \ldots ; y_{\mathrm{i}} ; \ldots ; y_{p}\right]$, where

$$
P_{\text {in }, \text { out }(\bmod )}= \begin{cases}0 & t \in[0 ; T) \\ \vdots & \\ x(t), y(t) & t \in[(i-1) T ; i T) \\ 0 & t \in[i T ;(i+1) T) \\ \vdots & \\ 0 & t \in[(p-1) T ; p T) .\end{cases}
$$

TABLE I: THE PARAMETERS OF THE MICROBUBBLES

\begin{tabular}{ccc}
\hline Symbol & Description & Value \\
\hline$R_{0}$ & Equilibrium radius of the bubble $(\mathrm{m})$ & $1.5 \times 10^{-6}$ \\
$d_{s}$ & Shell thickness of the bubble $(\mathrm{m})$ & $0.1 \% \times R_{0}$ \\
$G_{s}$ & Shear modulus of the shell $(\mathrm{MPa})$ & 10 \\
$\rho_{L}$ & Density of water $\left(\mathrm{kg} \bullet \mathrm{m}^{-3}\right)$ & 1000 \\
$p_{0}$ & Hydrostatic pressure $(\mathrm{Pa})$ & $1.01 \times 10^{5}$ \\
$\mu_{L}$ & Shear viscosity of water $(\mathrm{Pa} \bullet \mathrm{s})$ & 0.001 \\
$\mu_{s}$ & Shear viscosity of the shell $(\mathrm{Pa} \bullet \mathrm{s})$ & 1.49 \\
$\kappa$ & Polytropic exponent & 1.09 \\
\hline
\end{tabular}

It is easy to verify that

$$
\begin{aligned}
x_{1}(t)+\ldots+x_{i}(t)+\ldots+x_{p}(t) & =x(t) \\
x_{i}(t) & =x_{1}(t-i T)
\end{aligned}
$$

and also

$$
\begin{aligned}
y_{1}(t)+\ldots+y_{i}(t)+\ldots+y_{p}(t) & =y(t) \\
y_{i}(t) & =y_{1}(t-i T) .
\end{aligned}
$$

Fig. 1 shows the block diagram of Volterra series kernel identification using the simulation data generated from microbubble model. After identification, all coefficients of the zero-order, first-order, second-order, and third-order Volterra kernels are determined. Then, the estimated subharmonic signal can be determined from the third-order MISO Volterra series according to the block diagram shown in Fig. 2. The input $P_{i n}$ is modified to be $P_{i n(\bmod )}$ with a time multiplexer $M$. The output from each subsystem 资 $y_{2}, \ldots, y_{p}$ is calculated and summed to the estimated output $\hat{y}(t)$.

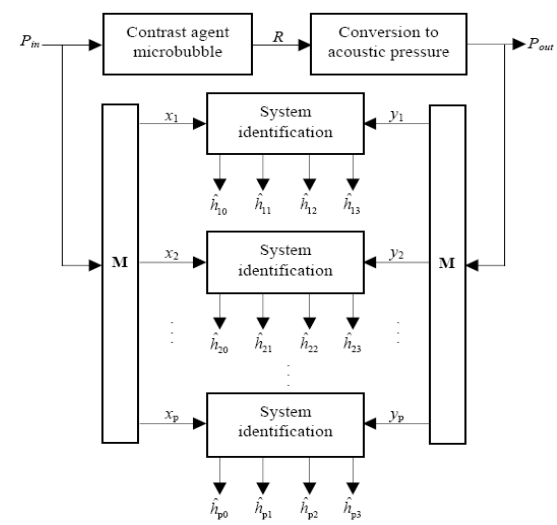

Fig. 1. Identification of MISO Volterra series from the microbubble model.

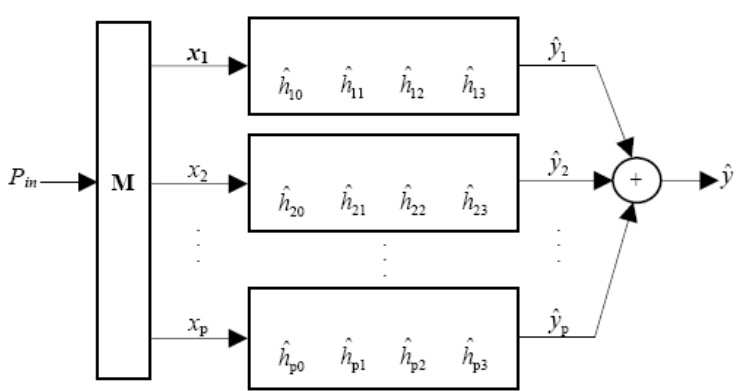

Fig. 2. Structure of MISO Volterra series modeling for subharmonic system. 


\section{Performance Evaluation}

To evaluate performance of the model order and system memory, we compute the normalized mean-square error (NMSE) as follows:

$$
\mathrm{NMSE}=\frac{\left\|\hat{y}_{\text {Volterra }}-y_{\text {Church }}\right\|_{2}}{\left\|y_{\text {Church }}-\bar{y}_{\text {Church }}\right\|_{2}},
$$

where $\hat{y}_{\text {Volterra }}$ denotes the predicted output from Volterra series, $y_{\text {Church }}$ denotes the output obtained from the simulation of the Church equation, and $\bar{y}_{\text {Church }}$ denotes the mean value of the output. The operator $\|\cdot\|_{2}$ denotes the $l_{2}$ norm.

Various experimental conditions are performed and evaluated. The excitation amplitude values consists of three pressure ranges, i.e. 0.1 $\mathrm{MPa}$ (low), 0.6 $\mathrm{MPa}$ (medium), and 1.2 $\mathrm{MPa}$ (high). Furthermore, the length of system memory was varied from 2 to 22 samples in steps of 2 .

\section{RESUlTS AND DISCUSSION}

\section{A. Simulation of Subharmonic generation}

In order to estimate the values of coefficients from the MISO Volterra model, we use the predicted output in which the modified input-output signals are obtained by using (15-17b). Fig. 3-5 shows the predicted output for excitation pressures of $0.1 \mathrm{MPa}, 0.6 \mathrm{MPa}$ and $1.2 \mathrm{MPa}$. The results from comparison between input and output signals show that the predicted output consisting of subharmonics has a period two times larger than that of the original input.

Next step, the original input-output signals are decomposed to the input components $x_{1}^{\text {new }}$ and $x_{2}^{\text {new }}$ and the output components $y_{1}^{\text {new }}$ and $y_{2}^{\text {new }}$. The input and output signals after decomposition with excitation pressures of 0.1 , 0.6, and 1.2 MPa are shown in Fig. 6(a)-(b), Fig. 7(a)-(b), and Fig.8(a)-(b), respectively. Then, the components of input $\left[x_{1}^{\text {new }} ; x_{2}^{\text {new }}\right]$ and output $\left[y_{1}^{\text {new }} ; y_{2}^{\text {new }}\right]$ were used for finding the coefficients of MISO Volterra model. After the coefficients are identified, the third-order MISO Volterra model for subharmonic system can be expressed as

$$
\begin{gathered}
\hat{y}_{1}(n)=\hat{h}_{10}+\sum_{\tau_{1}=0}^{N-1} \hat{h}_{11}\left(\tau_{1}\right) x_{1}\left(n-\tau_{1}\right) \\
+\sum_{\tau_{1}=0}^{N-1} \sum_{\tau_{2}=0}^{N-1} \hat{h}_{12}\left(\tau_{1}, \tau_{2}\right) x_{1}\left(n-\tau_{1}\right) x_{1}\left(n-\tau_{2}\right) \\
+\sum_{\tau_{1}=0}^{N-1} \sum_{\tau_{2}=0}^{N-1} \sum_{\tau_{3}=0}^{N-1} \hat{h}_{13}\left(\tau_{1}, \tau_{2}, \tau_{3}\right) x_{1}\left(n-\tau_{1}\right) x_{1}\left(n-\tau_{2}\right) x_{1}\left(n-\tau_{3}\right)
\end{gathered}
$$

$$
\begin{gathered}
\hat{y}_{2}(n)=\hat{h}_{20}+\sum_{\tau_{1}=0}^{N-1} \hat{h}_{21}\left(\tau_{1}\right) x_{2}\left(n-\tau_{1}\right) \\
+\sum_{\tau_{1}=0}^{N-1} \sum_{\tau_{2}=0}^{N-1} \hat{h}_{22}\left(\tau_{1}, \tau_{2}\right) x_{2}\left(n-\tau_{1}\right) x_{2}\left(n-\tau_{2}\right) \\
+\sum_{\tau_{1}=0}^{N-1} \sum_{\tau_{2}=0}^{N-1} \sum_{\tau_{3}=0}^{N-1} \hat{h}_{23}\left(\tau_{1}, \tau_{2}, \tau_{3}\right) x_{2}\left(n-\tau_{1}\right) x_{2}\left(n-\tau_{2}\right) x_{2}\left(n-\tau_{3}\right)
\end{gathered}
$$

where

$$
\hat{y}(n)=\hat{y}_{1}(n)+\hat{y}_{2}(n)
$$

The MISO model in (21) is corresponding to the predicted output as the structure given in Fig. 2.
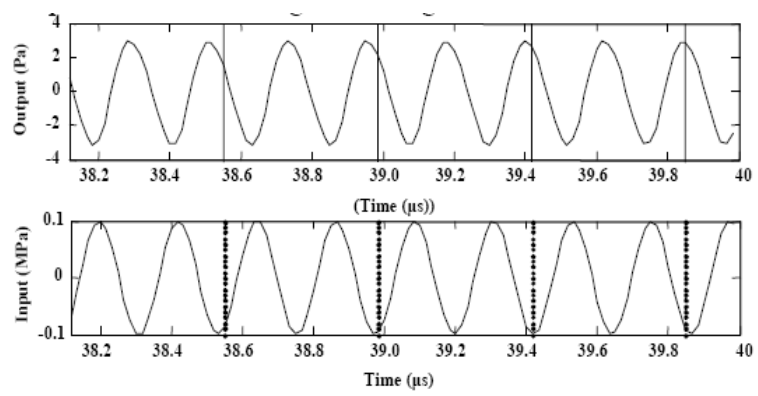

Fig. 3. Predicted output signal for $0.1 \mathrm{MPa}$ excitation pressure from the Church's equation compared with the input signal.
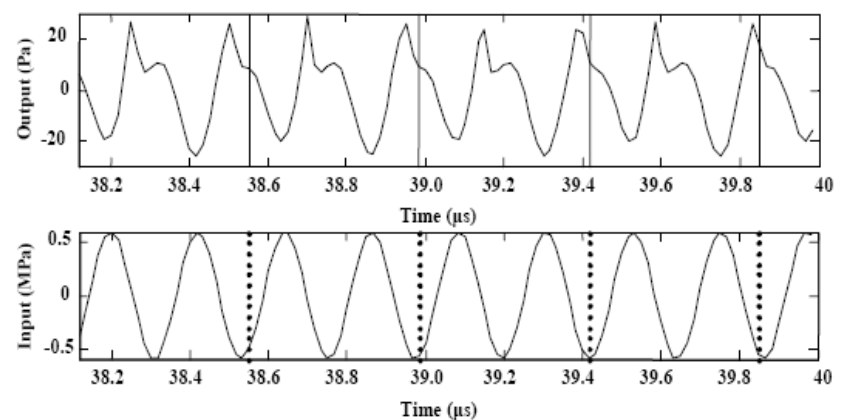

Fig. 4. Predicted output signal for $0.6 \mathrm{MPa}$ excitation pressure from the Church's equation compared with the input signal.
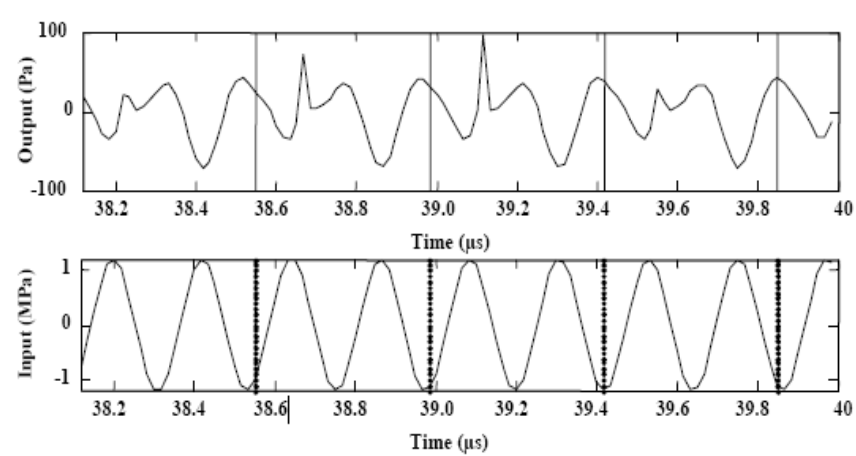

Fig. 5. Predicted output signal for 1.2 MPa excitation pressure from the Church's equation compared with the input signal. 

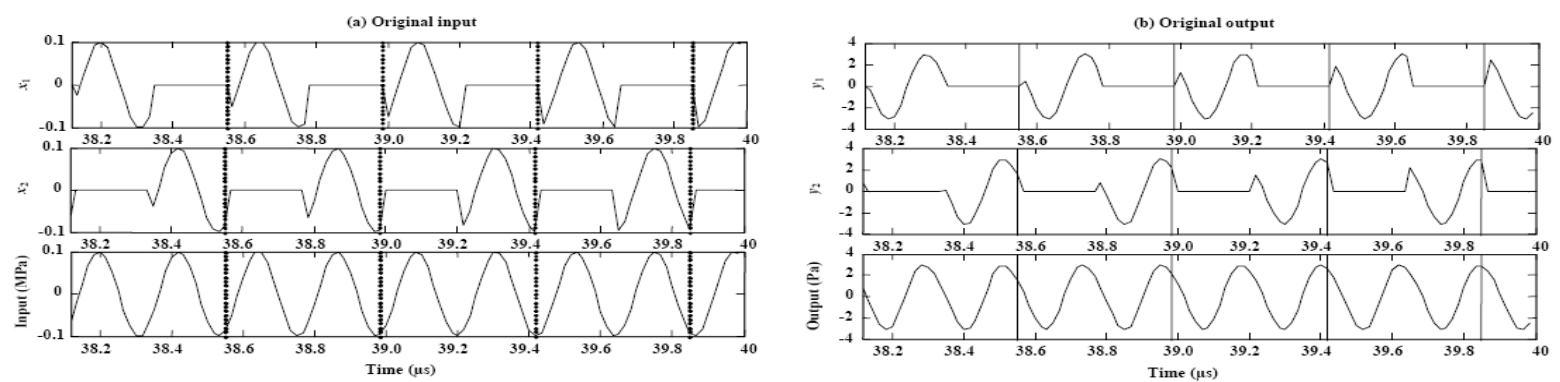

Fig. 6. Modified input and output signals for 0.1 MPa excitation pressure: (a) input; (b) output.
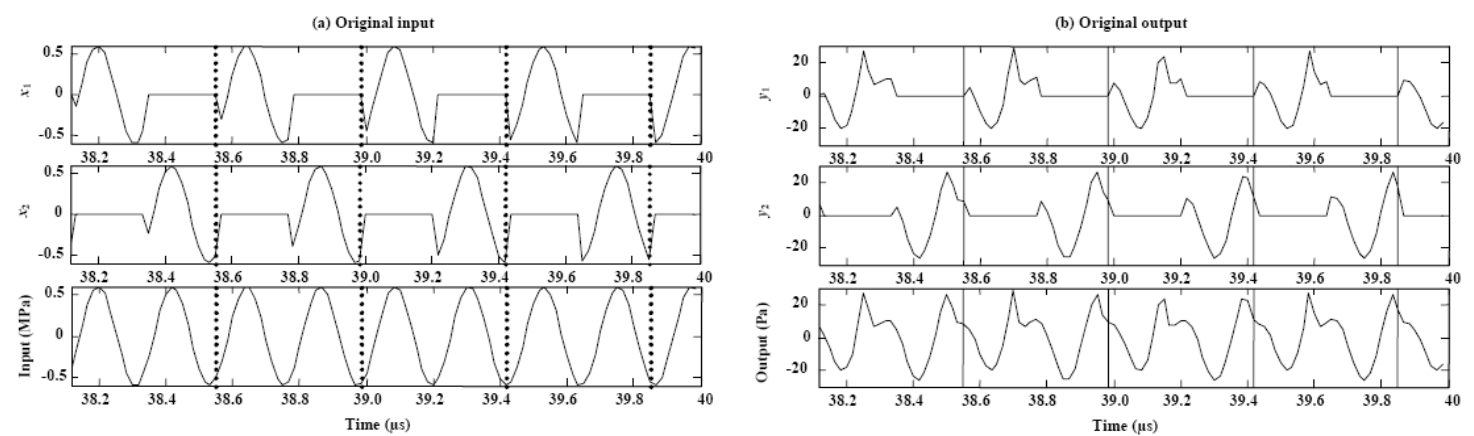

Fig. 7. Modified input and output signals for $0.6 \mathrm{MPa}$ excitation pressure: (a) input; (b) output.
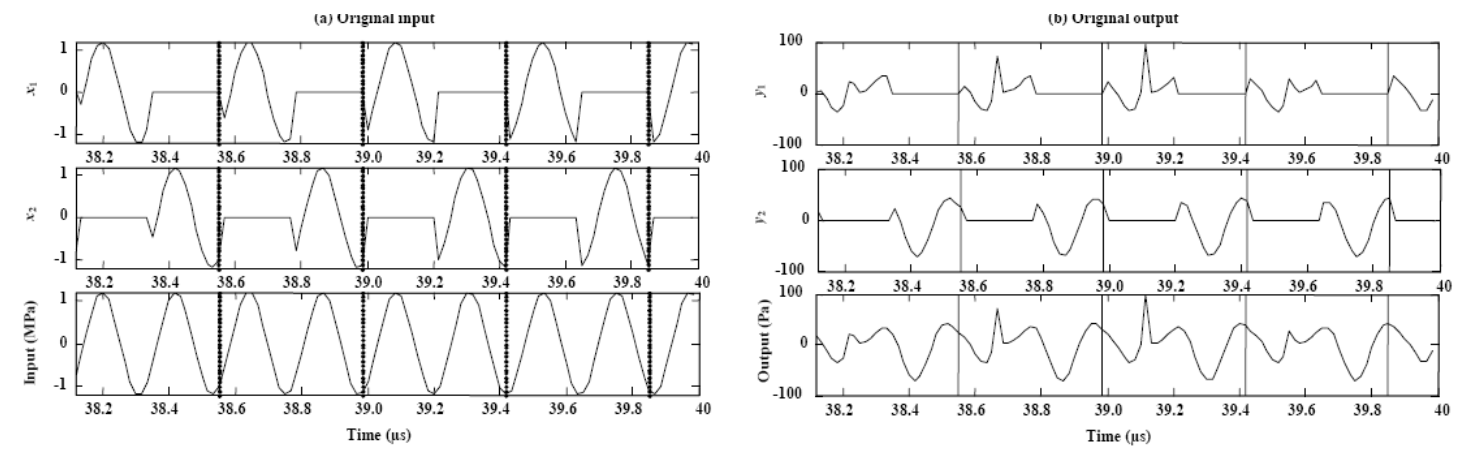

Fig. 8. Modified input and output signals for 1.2 MPa excitation pressure: (a) input; (b) output.

\section{B. System Memory}

The NMSE results for the excitation pressure of $0.1 \mathrm{MPa}$, $0.6 \mathrm{MPa}$, and 1.2 $\mathrm{MPa}$ obtained by varying the length of system memory from 2 to 22 are shown in Fig. 9-11.

At $0.1 \mathrm{MPa}$ of excitation pressure (Fig. 9), there is a small difference for the NMSE in the range of 2-16 sample length of system memory. The NMSE performance of the first-order MISO Volterra series with the length of 22 sample system memory is $-11.21 \mathrm{~dB}$. When the orders increase to the second and the third, the NMSE values decrease to $-18.04 \mathrm{~dB}$ and $-30.48 \mathrm{~dB}$, respectively.

For an excitation pressure of $0.6 \mathrm{MPa}$ (Fig. 10), the memory length of 22 samples provides the best achievable

NMSE from the first-order Volterra series at $-3.97 \mathrm{~dB}$. The second-order and third-order terms improve the attainable NMSE to $-8.16 \mathrm{~dB}$ and $-12.01 \mathrm{~dB}$, respectively.

For an excitation pressure of 1.2 MPa (Fig. 11), significant difference in NMSE values for first-order second-order and third-order Volterra series can be seen. This is due to the fact that the nonlinearity of the microbubble becomes apparent at the high excitation pressure. At a memory length of 22 samples, the NMSE values of first-order, second-order and third-order terms are $-2.95 \mathrm{~dB},-5.16 \mathrm{~dB}$ and $-6.52 \mathrm{~dB}$, respectively.

\section{Responses of MISO Volterra Series Model}

In this section, we demonstrate a capability of a third-orderVolterra MISO model to estimate the oscillation behavior of microbubble in both time and frequency domains. The estimated first-, second- and third-order Volterra models were tested with excitation pressure inputs of $0.1,0.6$ and 1.2 MPa. The first-order model includes only the linear operator while the second-order model also includes both the linear and quadratic operator, and third-order Volterra model includes the linear, second and third operator. The length of system memory $N=22$ was used in kernel estimation.

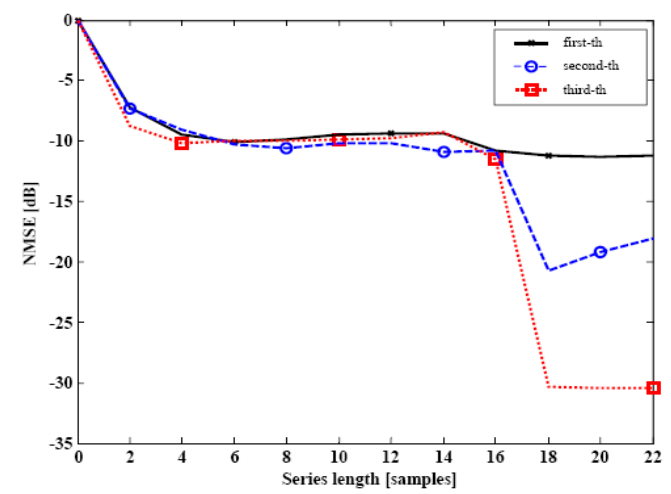

Fig. 9. NMSE as a function of system memory for 0.1 MPa excitation pressure resulting from the first-, second- and third-order MISO Volterra series. 


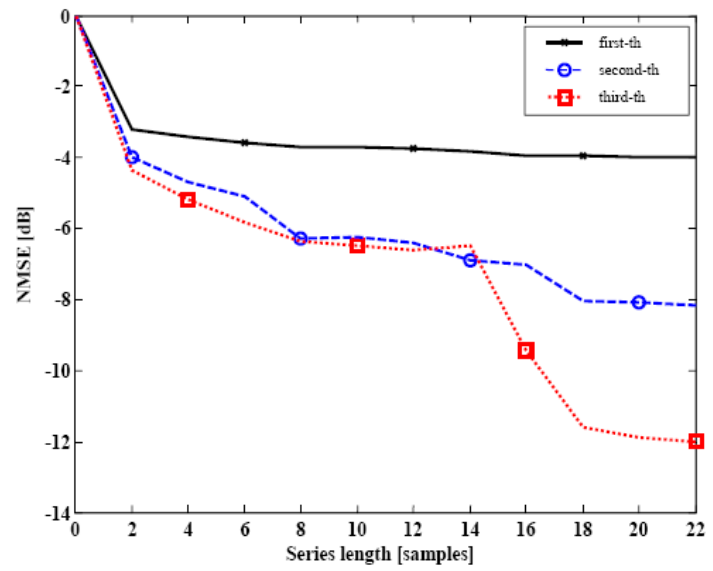

Fig. 10. NMSE as a function of system memory for $0.6 \mathrm{MPa}$ excitation pressure resulting from the first-, second- and third-order MISO Volterra series.

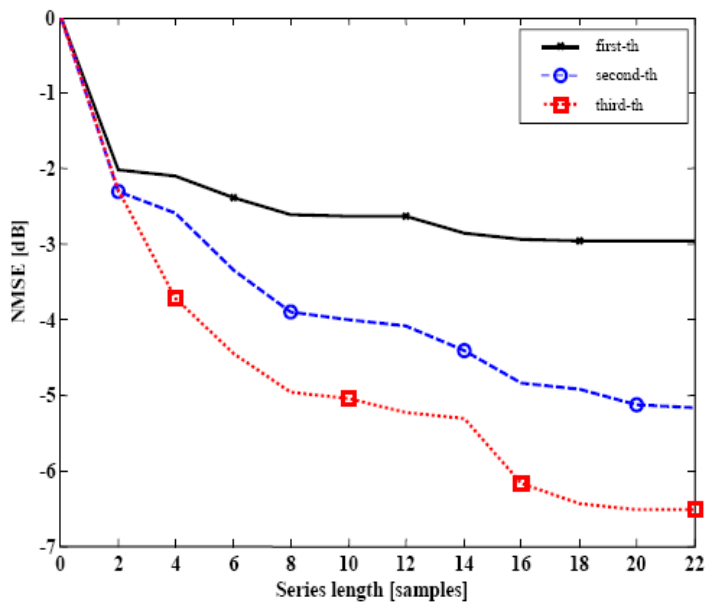

Fig. 11. NMSE as a function of system memory for 1.2 MPa excitation pressure resulting from the first-, second- and third-order MISO Volterra series.

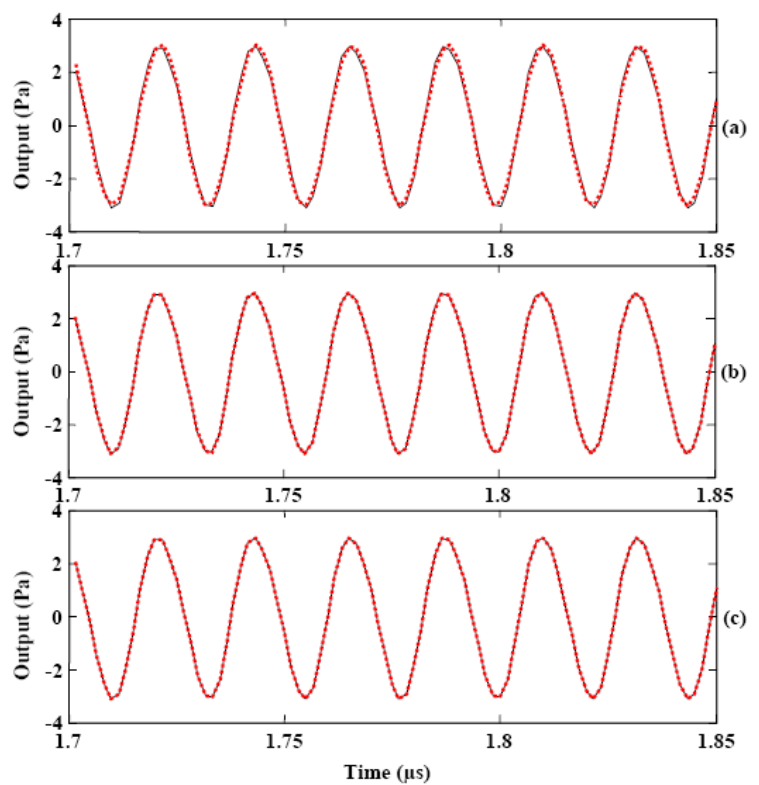

Fig. 12. (a) Fist-order output response (b) up to the second-order response and (c) up to the third-order response, Church (solid line) and Volterra model (dashed line). The excitation amplitude pressure is $0.1 \mathrm{MPa}$.
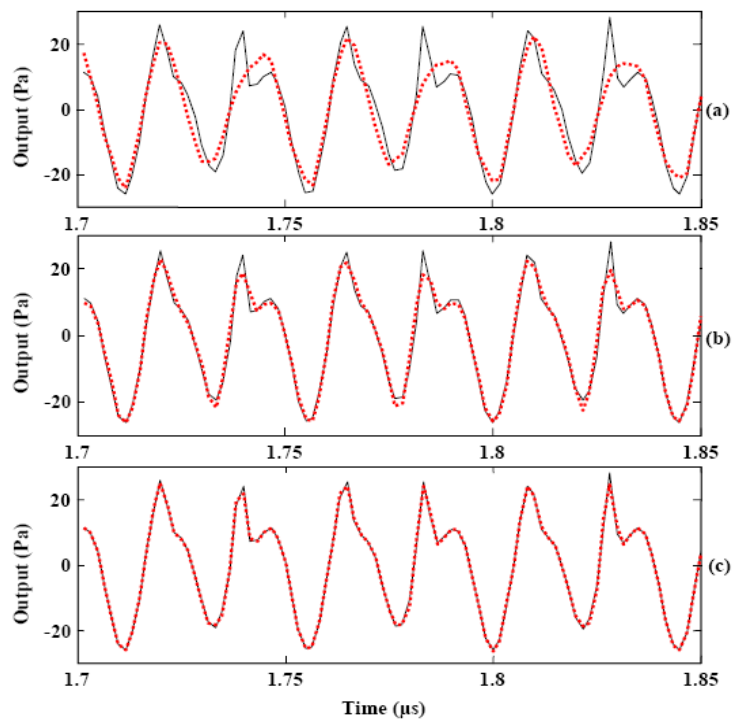

Fig. 13. (a) Fist-order output response (b) up to the second-order response and (c) up to the third-order response, Church (solid line) and Volterra model (dashed line). The excitation amplitude pressure is 0.6 MPa.

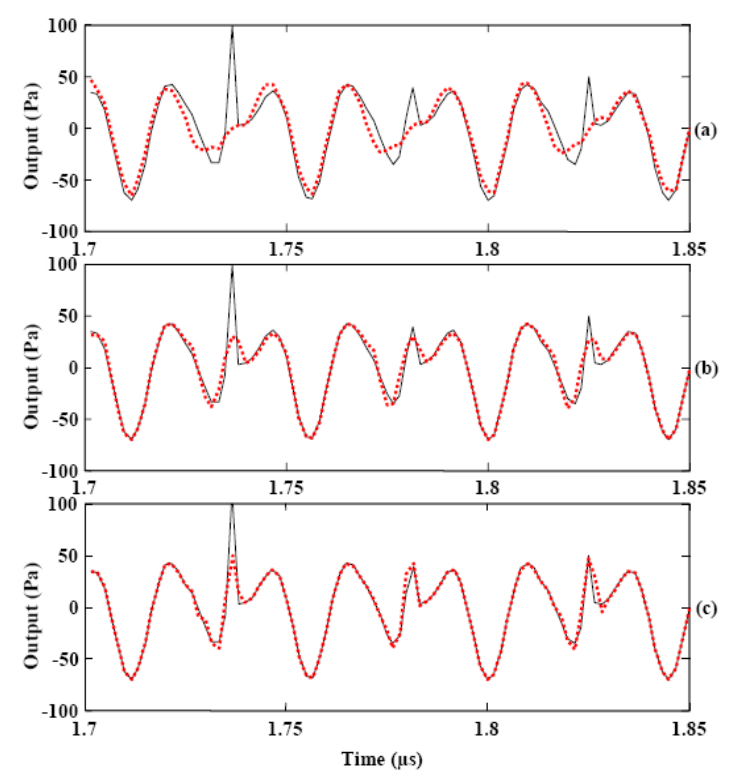

Fig. 14. (a) Fist-order output response (b) up to the second-order response and (c) up to the third-order response, Church (solid line) and Volterra model (dashed line). The excitation amplitude pressure is 1.2 MPa.

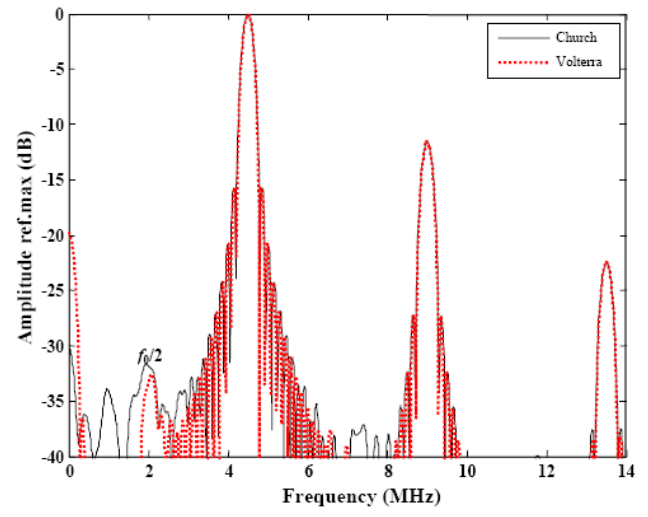

Fig. 15. Response of Church (solid line) and third-order Volterra model (dashed line) for the excitation amplitude pressure at $0.1 \mathrm{MPa}$. Subharmonic generation is weak. 
The comparisons between the outputs from Church's equation and the MISO models are shown in Fig. 12-14. The results show that the predicted outputs from the third-order Volterra can achieve better approximation compared to those from the first-order and the second-order Volterra for all amplitude input cases of $0.1,0.6$ and $1.2 \mathrm{MPa}$ as shown in Fig. 12(c), 13(c) and 14(c), respectively. The corresponding results can be seen in the frequency domain between the spectra of signals from Church's model and the third-order Volterra models as shown in Fig. 15, Fig. 16 and Fig. 17, respectively.

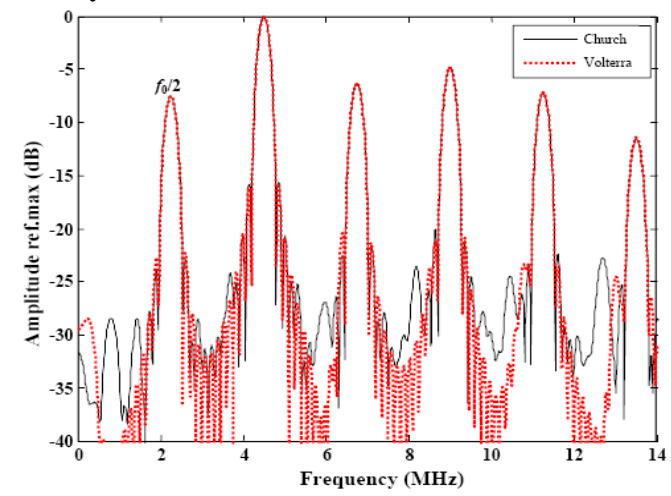

Fig. 16. Response of church (solid line) and third-order volterra model (dashed line) for the excitation amplitude pressure at $0.6 \mathrm{MPa}$. subharmonic generation is growth.

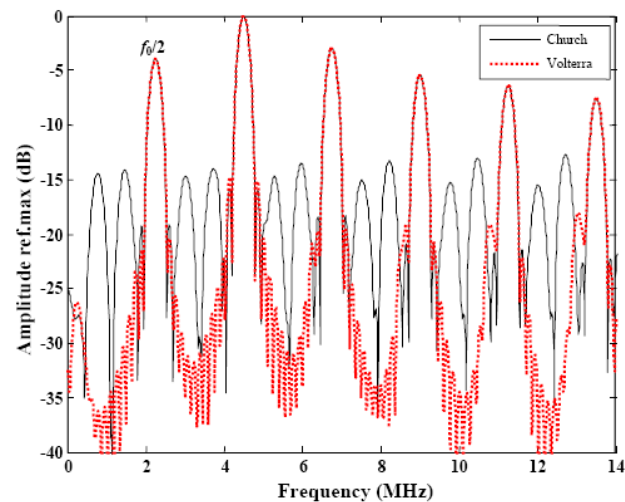

Fig. 17. Response of church (solid line) and third-order volterra model (dashed line) for the excitation amplitude pressure at 1.2 MPa. subharmonic generation is saturation.

\section{CONCLUSIONS AND DisCUSSION}

In this work, we have presented the modeling the oscillation behavior of subharmonics of ultrasound contrast agent by using MISO Volterra model. The proposed method employs the modification of input-output signals for identifying the kernels of the third-order Volterra model. The MISO method has been successfully illustrated in modeling subharmonic signals from the numerical solution of Church's equation. The results show that the predicted output from the third-order MISO Volterra can accurately approximate the responses from the Church's equation both in time domain and frequency domain.

\section{ACKNOWLEDGMENT}

This work is in part supported by Department of Electrical Engineering, Faculty of Engineering, Prince of Songkla University.

\section{REFERENCES}

[1] F. Calliada, R. Campani, O. Bottinelli, A. Bozzini and M. G. Sommaruga, "Ultrasound contrast agents basic principles," Eur. J. Radial., vol. 27, pp. S157-S160, 1998.
[2] D. Cosgrove, "Ultrasound contrast agents: An overview," Eur. J. Radial, vol. 60, pp. 324-330, 2006.

[3] N. D. Jong, M. Emmer, A. V. Wamel, and M. Versluis, "Ultrasonic characterization of ultrasound contrast agents," Med. Biol. Eng. Comput, vol. 47, pp. 861-873, 2009.

[4] V. Sboros, "Response of contrast agents to ultrasound," Adv. Drug Deliv. Rev, vol. 60, pp. 1117-1136, 2008

[5] F. Forsberg, D. A. Merton, J. B. Liu, L. Needleman, and B. B. Goldberg, "Clinical applications of ultrasound contrast agents," Ultrasonics, vol. 36, pp. 695-701, 1998.

[6] P. M. Shankar, P. D. Krishna, and V. L. Newhouse, "Advantages of subharmonic over second harmonic backscatter for contrast-to-tissue echo enhancement," Ultrasound Med. Biol, vol. 24, pp. 395-399, 1998.

[7] F. Forsberg, W. T. Shi, and B. B. Goldberg, "Subharmonic imaging of contrast agents," Ultrasonics, vol. 38, pp. 93-98, 2000.

[8] F. Forsberg, C. W. Piccoli, D. A. Merton, J. J. Palazzo, and A. L. Hall, "Breast lesions: Imaging with contrast-enhanced subharmonic US-initial experience," Radiology, vol. 244, pp. 718-726, 2007.

[9] P. D. Krishna, P. M. Shankar, and V. L. Newhouse, "Subharmonic generation from ultrasonic contrast agents," Phys. Med. Biol., vol. 44, pp. 681-694, 1999.

[10] A. Katiyar and K. Sarkar, "Modeling subharmonic response from contrast microbubbles as a function of ambient static pressure," $J$. Acoust. Soc. Am, vol.129, no. 4, pp. 2325-2335, April 2011

[11] G. Y. Jun, Z. Dong, G. X. Fen, T. K. Bin, and L. Zheng, "The viscoelasticity of lipid shell and the hysteresis of subharmonic in liquid containing microbubbles," Chin. Phys. Soc, vol. 15, no. 7, pp 1526-1531, July 2006.

[12] M. Mleczko, M. Postema, and G. Schmitz, "Discussion of the application of finite Volterra series for the modeling of the oscillation behavior of ultrasound contrast agents," Appl. Acoust, vol. 70, pp. 1363-1369, 2009.

[13] P. Phukpattaranont and E. S. Ebbini, "Post beamforming second-order Volterra filter for pulse echo ultrasonic imaging," IEEE Trans. Ultrason, Ferroelect, Freq Contr, vol. 50, no. 8, pp. 987-1001, 2003.

[14] O. M. Boaghe and S. Billings, "Subharmonic oscillation modeling and MISO Volterra series," IEEE Trans. Circ. Syst. Fund. Theor. Appl, vol. 50, no. 7, pp. 877-884, July 2003.

[15] L. M. Li and S. A. Billings, "Discrete time subharmonic modelling and analysis," International Journal of Control, vol. 78, pp. 1265-1284, 2005.

[16] W. J. Rugh, "Nonlinear System Theory: The Volterra Wiener Approach," Johns Hopkins University Press, 1981, 1-2.

[17] M. Schetzen, "The Volterra and Wiener Theories of Nonlinear Systems," New York: Wiley, 1980, pp. 1-3.

[18] Regularization Tool, Per Christian Hansen, Department of Mathematical Modelling, Technical University of Denmark, 1998, pp. 7-29.

[19] C. Samakee and P. Phukpattaranont, "Simulation of subharmonic generation based on nonlinear oscillation from ultrasound contrast agents," in Proc. 8th ECTI Conf. Signal Processing, Khon Kaen, 2011 pp. 1015-1018.

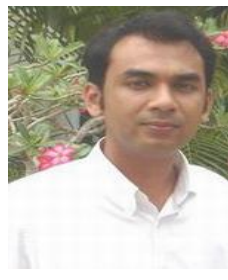

C. Samakee Chinda Samakee received B.Eng. degree from Siam University in 1996 and M. Eng. degree in Electrical Engineering from Prince of Songkla University in 2004. He is currently working toward his Ph.D. degree in Electrical Engineering at Prince of Songkla University. His research interests include ultrasound signal processing, ultrasound contrast imaging, and signal processing

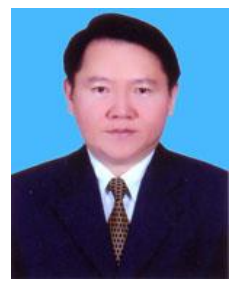

P. Phukpattaranont was born in Songkla, Thailand He received the B. Eng. and M. Eng. degrees in electrical engineering from Prince of Songkla University in 1993 and 1997, respectively, the Ph.D. degree in Electrical and Computer Engineering from the University of Minnesota, in 2004. He is currently an assistant professor of Electrical Engineering at Prince of Songkla University. His research interests include ultrasound contrast imaging, signal processing, image processing, and biomedical instrumentation. Dr. Phukpattaranont is a member of the IEEE 